\title{
Levi Factors and Admissible Automorphisms
}

\author{
Meng-Kiat Chuah $^{1} \cdot$ Rita Fioresi $^{2}$ (D)
}

Received: 15 April 2020 / Accepted: 27 December 2020 / Published online: 1 March 2021

(C) The Author(s) 2021

\begin{abstract}
Let $\mathfrak{g}$ be a complex simple Lie algebra. We consider subalgebras $\mathfrak{m}$ which are Levi factors of parabolic subalgebras of $\mathfrak{g}$, or equivalently $\mathfrak{m}$ is the centralizer of its center. We introduced the notion of admissible systems on finite order $\mathfrak{g}$-automorphisms $\theta$, and show that $\theta$ has admissible systems if and only if its fixed point set is a Levi factor. We then use the extended Dynkin diagrams to characterize such automorphisms, and look for automorphisms of minimal order.
\end{abstract}

Keywords Complex simple Lie algebra · Automorphism · Levi factor ·

Admissible system · Dynkin diagram

Mathematics Subject Classification (2010) 17B20 $\cdot 17 \mathrm{~B} 22 \cdot 17 \mathrm{~B} 40$

\section{Introduction}

Let $\mathfrak{g}$ be a finite dimensional complex simple Lie algebra. An involution $\theta$ on $\mathfrak{g}$ leads to the decomposition $\mathfrak{g}=\mathfrak{k}+\mathfrak{p}$, consisting of the \pm 1 -eigenspaces of $\theta$. It is well-known that $\mathfrak{k}$ has a 1-dimensional center if and only if there exists a positive system of roots such that $\mathfrak{p}=\mathfrak{p}^{+}+\mathfrak{p}^{-}$, and $\mathfrak{k}$ acts irreducibly on $\mathfrak{p}^{ \pm}$. Such systems are called admissible and they play a key role in the construction of the holomorphic Harish-Chandra representations of a simple real group (see $[2,6])$.

One may generalize $\mathfrak{k}+\mathfrak{p}^{+}$to a general parabolic subalgebra $\mathfrak{m}+\mathfrak{n}$ of $\mathfrak{g}$, where $\mathfrak{m}$ is reductive and $\mathfrak{n}$ is the nilradical. Here $\mathfrak{m}$ is called a Levi factor of parabolic subalgebra, and Kostant [9] studied the restriction of its root system to the center of $\mathfrak{m}$ with the Borel-de

\footnotetext{
Presented by: Vyjayanthi Chari

Rita Fioresi

rita.fioresi@unibo.it

Meng-Kiat Chuah

chuah@math.nthu.edu.tw

1 Department of Mathematics, National Tsing Hua University, Hsinchu 300, Taiwan

2 Dipartimento di Matematica, University of Bologna, Piazza Porta San Donato 5, 40126 Bologna, Italy
} 
Siebenthal property, which led to a nice description of the $\mathfrak{m}$-representation on $\mathfrak{n}$. In view of the $\mathfrak{k}+\mathfrak{p}$ setting and Kostant's work, in this article, we incorporate higher order automorphisms $\theta$ and admissible positive systems in the study of $\mathfrak{m}$. We also characterize them by the extended Dynkin diagram of $\mathfrak{g}$.

Our work establishes a correspondence between certain gradings of $\mathfrak{g}$ and the $\mathfrak{g}$ automorphisms with admissible positive systems. The existence of such simple systems in the classical theory (see [6]) is crucial in the construction of the Harish-Chandra representations of a given real form $G_{\mathbb{R}}$ of $G$, the simply connected group with $\mathfrak{g}=\operatorname{Lie}(G)$, when $\operatorname{rank} \mathfrak{k}=$ rank $\mathfrak{g}$. Such representations are geometrically realized in [6] in the space of holomorphic sections of suitable line bundles in $G_{\mathbb{R}} / K_{\mathbb{R}}$, where $K_{\mathbb{R}}$ is a maximal compact subgroup of $G_{\mathbb{R}}$. The infinitesimal theory by Kostant in [9] treats a generalized notion of root systems, where the role of Cartan subalgebra is replaced by a non-maximal toral subalgebra. Our broader notion of admissible systems then appears naturally in this context (see Sec. 2.3 in [9]) and suggests future applications to the theory of root systems and representations, where $\mathfrak{k}$ is replaced by $\mathfrak{g}^{\theta}$ for an admissible $\theta$.

We also briefly mention that, $\mathbb{Z}_{3}$ and $\mathbb{Z}_{5}$ gradings of $\mathfrak{g}=E_{7}$ appear naturally in supergravity, leading to a generalization of Freudenthal magic square (see [5, 10]), involving the realization of $G$ as the conformal group over $\mathbb{R}, \mathbb{C}, \mathbb{H}, \mathbb{O}$ (where $\mathbb{H}$ denotes the quaternions, (1) the octonions). These groups appear in $N>2$-extended $D=4$ supergravity theories with symmetric scalar manifolds and are essential to construct the so-called "exceptional hidden symmetries".

We now discuss our project in more details. We shall show that a subalgebra $\mathfrak{m}$ is Levi if and only if $\mathfrak{m}$ is the centralizer of its center (Theorem 2.3). It enables us to work with $\mathfrak{m}$ without referring to specific parabolic subalgebras. We shall study them as fixed points of $\mathfrak{g}$-automorphisms $\theta$. We say that $\theta$ is of equal rank if $\left.\theta\right|_{\mathfrak{h}}=1$ for some Cartan subalgebra $\mathfrak{h}$. This definition is motivated by the fact that the invariant subalgebra $\mathfrak{g}^{\theta}$ has the same rank as $\mathfrak{g}$. The condition $\left.\theta\right|_{\mathfrak{h}}=1$ implies that every root space $\mathfrak{g}_{\alpha}$ consists of eigenvectors of $\theta$.

We recall a theorem of Kac, which classifies all finite order $\mathfrak{g}$-automorphisms. A collection of nonnegative integers is said to be relatively prime if \pm 1 are their only common factors. Let $\mathrm{D}$ be the extended Dynkin diagram of $\mathfrak{g}$. Its vertices $\alpha$ represent some roots of $\mathfrak{h}$. There are canonical relatively prime positive integers $\left\{a_{\alpha}\right\}_{\mathrm{D}}$, known as labels, such that $\sum_{\mathrm{D}} a_{\alpha} \alpha=0$ [7, Ch.4]. A Kac diagram $k$ is an assignment of relatively prime nonnegative integers $\left\{k_{\alpha}\right\}_{\mathrm{D}}$. We say that $k$ represents an equal rank $\mathfrak{g}$-automorphism $\theta$ of order $s=\sum_{\mathrm{D}} a_{\alpha} k_{\alpha}$ if for all $\alpha \in \mathrm{D}$,

$$
\left.\theta\right|_{\mathfrak{g}_{\alpha}}=\omega^{k_{\alpha}}, \quad \text { where } \omega=\exp (2 \pi i / s) \in \mathbb{C} .
$$

Theorem 1.1 (Kac [7, Thm.8.6, Prop.8.6]) Up to equivalence, (1.1) provides a bijective correspondence between the Kac diagrams $k$ on $\mathrm{D}$ and the equal rank finite order $\mathfrak{g}$ automorphisms $\theta$. Here $\mathfrak{g}^{\theta}$ is reductive, its semisimple part has Dynkin diagram $\{\alpha \in$ $\left.\mathrm{D} ; k_{\alpha}=0\right\}$ and its center has dimension $\sharp\left\{\alpha \in \mathrm{D} ; k_{\alpha}>0\right\}-1$.

More precisely, given a Kac diagram $k$, we can immediately define an equal rank automorphism $\theta$ of order $s$ as:

$$
\left.\theta\right|_{\mathfrak{g}_{\alpha}}=\left\{\begin{array}{cl}
\omega^{k_{\alpha}} & \text { for } \alpha \in \mathrm{D} \backslash \operatorname{ker} k, \quad \omega=\exp (2 \pi i / s) \\
1 & \text { for } \alpha \in \operatorname{ker} k .
\end{array}\right.
$$

In Theorem 1.1, the equivalence refers to $k, k^{\prime}$ which are related by diagram symmetries of $\mathrm{D}$, as well as $\theta, \theta^{\prime}$ which are conjugated by $\mathfrak{g}$-automorphisms. The full result of Kac's 
theorem also includes finite order automorphisms which are not equal rank, and in that case the Kac diagrams are defined on some other diagrams (see Tables Aff 2, Aff 3 of [7, Ch.4]).

Let $\theta$ be a finite order $\mathfrak{g}$-automorphism of order $s$ of equal rank type. We have

$$
\mathfrak{g}=\sum_{\mathbb{Z}_{s}} \mathfrak{g}_{m}, \mathfrak{g}_{m}=\text { eigenspace of } \exp (2 \pi i m / s) \text { under } \theta .
$$

The equal rank condition implies that $\theta$ stabilizes each root space of $\mathfrak{h}$, so each $\mathfrak{g}_{m}$ is a sum of root spaces. We have $\left[\mathfrak{g}_{j}, \mathfrak{g}_{m}\right] \subset \mathfrak{g}_{j+m}$, so in particular $\mathfrak{g}_{0}$ acts on each $\mathfrak{g}_{m}$. Write $\mathfrak{g}^{\theta}=\mathfrak{g}_{0}$ for convenience. A positive system on $\mathfrak{h}$ leads naturally to the sum $\mathfrak{g}_{m}=\mathfrak{g}_{m}^{+}+\mathfrak{g}_{m}^{-}$. We say that the positive system is admissible for $\theta$ if for all $j, m \in \mathbb{Z}_{S} \backslash\{0\}$,

(a) $\left[\mathfrak{g}^{\theta}, \mathfrak{g}_{m}^{ \pm}\right] \subset \mathfrak{g}_{m}^{ \pm}$,

(b) $\left[\mathfrak{g}_{j}^{ \pm}, \mathfrak{g}_{m}^{ \pm}\right] \cap \mathfrak{g}^{\theta}=0$.

Note that when $s=2$, this is the familiar $\left[\mathfrak{k}, \mathfrak{p}^{ \pm}\right] \subset \mathfrak{p}^{ \pm}$and $\left[\mathfrak{p}^{ \pm}, \mathfrak{p}^{ \pm}\right]=0$. If $\theta$ has an admissible positive system, we say that $\theta$ is admissible. The admissible automorphisms are related to Levi factors by the next theorem.

Theorem 1.2 For each Levi factor $\mathfrak{m}$, there exists an admissible automorphism $\theta$ such that $\mathfrak{m}=\mathfrak{g}^{\theta}$. Conversely, if $\theta$ is an admissible automorphism, then $\mathfrak{g}^{\theta}$ is a Levi factor.

The next theorem classifies all admissible automorphisms. We say that a Kac diagram $k$ is relatively prime if $\left\{a_{\alpha} ; k_{\alpha}>0\right\}$ are relatively prime. In particular if $k_{\alpha}>0$ for some $a_{\alpha}=1$, we say that $k$ is preferred. Thus a preferred Kac diagram is relatively prime, and the converse is true for classical Lie algebras because $\left\{a_{\alpha}\right\}_{\mathrm{D}} \subset\{1,2\}$.

Theorem 1.3 Let $\theta$ be represented by a Kac diagram $k$ on $\mathrm{D}$. Then $\theta$ is admissible if and only if $k$ is relatively prime.

Two Levi factors $\mathfrak{m}, \mathfrak{m}^{\prime}$ are said to be strongly isomorphic, denoted by $\mathfrak{m} \cong \mathfrak{m}^{\prime}$, if there exists a $\mathfrak{g}$-automorphism $\sigma$ such that $\sigma(\mathfrak{m})=\mathfrak{m}^{\prime}$. The above theorems allow us to study Levi factors as fixed point sets $\mathfrak{m}=\mathfrak{g}^{\theta}$, where the Kac diagrams of $\theta$ are relatively prime. Clearly there are more $\theta$ than $\mathfrak{m}$, due to the possibility of $\mathfrak{g}^{\theta} \cong \mathfrak{g}^{\theta^{\prime}}$ for various $\theta, \theta^{\prime}$. It is natural to look for $\theta$ of minimal order. The next theorem says that such automorphisms arise from preferred Kac diagrams. As mentioned above, this theorem is meaningful only for exceptional Lie algebras.

Theorem 1.4 Let $\mathfrak{m} \subset \mathfrak{g}$ be a Levi factor. There exists a $\mathfrak{g}$-automorphism $\theta$ such that $\mathfrak{g}^{\theta}=$ $\mathfrak{m}$, and the Kac diagram of $\theta$ is preferred. Furthermore if $\theta^{\prime}$ is another $\mathfrak{g}$-automorphism such that $\mathfrak{g}^{\theta} \cong \mathfrak{g}^{\theta^{\prime}}$, then order $(\theta) \leq \operatorname{order}\left(\theta^{\prime}\right)$.

Most of our results are correct on simple Lie algebras over other algebraically closed fields of characteristic 0 . However, we state them for complex simple Lie algebras in order to compare with the results of existing literature.

The sections in this article are arranged as follows.

Section 2 proves Theorem 2.3.

Section 3 proves Theorem 1.2.

Section 4 proves Theorem 1.3.

Section 5 proves Theorem 1.4.

Section 6 provides some examples to illustrate the results. 


\section{Levi Factors}

Let $\mathfrak{g}$ be a complex simple Lie algebra. In this section, we prove Theorem 2.3 , which characterizes the Levi factors in terms of their centers. This theorem has been indirectly assumed in some articles (see for instance [3, 4] and [9, Rk.1.1]). For completeness we state and prove it here since we will use it later.

Let $\mathfrak{h} \subset \mathfrak{q} \subset \mathfrak{g}$, where $\mathfrak{q}$ is a subalgebra, and $\mathfrak{h}$ is a Cartan subalgebra of $\mathfrak{g}$ with root system $\Delta \subset \mathfrak{h}^{*}$. We have the root space decomposition

$$
\mathfrak{q}=\mathfrak{h} \oplus \sum_{\alpha \in Q} \mathfrak{g}_{\alpha},
$$

where $Q \subset \Delta$. We say that $Q$ is parabolic if

(a) If $\alpha, \beta \in Q$ and $\alpha+\beta \in \Delta$, then $\alpha+\beta \in Q$,

(b) $\Delta=Q \cup(-Q)$.

Then $\mathfrak{q}$ is a parabolic subalgebra of $\mathfrak{g}$ if and only if $Q$ is a parabolic subset of $\Delta$.

We say that $\mathfrak{m} \subset \mathfrak{g}$ is a Levi factor if it is a maximal reductive subalgebra of a (not unique) parabolic subalgebra $\mathfrak{q}$. Then $\mathfrak{m}$ contains a Cartan subalgebra $\mathfrak{h}$, and we write $\mathfrak{m}=$ $\mathfrak{h} \oplus \sum_{\alpha \in M} \mathfrak{g}_{\alpha}$ where $M \subset \Delta$. The parabolic subset $Q$ is equivalently described as

$$
Q=\Delta^{+} \coprod M^{-}
$$

where $\Delta^{+}$is a positive system and $M^{ \pm}=M \cap \Delta^{ \pm}$. See [1, Prop.7.20]. A subalgebra of $\mathfrak{g}$ is called toral if it is contained in a Cartan subalgebra of $\mathfrak{g}$.

Proposition 2.1 Suppose that the center $\mathfrak{z}$ of $\mathfrak{m}$ is toral, and $\mathfrak{m}$ is the centralizer of $\mathfrak{z}$ in $\mathfrak{g}$. Then $\mathfrak{m}$ is a Levi factor.

Proof Suppose that the center $\mathfrak{z}$ of $\mathfrak{m}$ is toral, and $\mathfrak{m}$ is the centralizer of $\mathfrak{z}$ in $\mathfrak{g}$. Then $\mathfrak{z}$ is contained in a Cartan subalgebra $\mathfrak{h}$ of $\mathfrak{g}$, and since $\mathfrak{h}$ centralizes $\mathfrak{z}$, we have $\mathfrak{h} \subset \mathfrak{m}$. Let $\mathfrak{g}_{\alpha}$ be a root space relative to $\mathfrak{h}$. We have $\mathfrak{g}_{\alpha} \subset \mathfrak{m}$ if and only if $\left.\alpha\right|_{\mathfrak{z}}=0$, so

$$
\mathfrak{m}=\mathfrak{h} \oplus \sum_{\left.\alpha\right|_{\mathfrak{z}}=0} \mathfrak{g}_{\alpha}
$$

Consider the linear subspace

$$
W=\left\{v \in \mathfrak{h}^{*} ; v(t)=0 \text { for all } t \in \mathfrak{z}\right\} .
$$

Fix $z \in \mathfrak{h}$ such that for all $\alpha \in \Delta, \alpha(z) \in \mathbb{R}$ and

$$
\alpha(z)=0 \Longleftrightarrow \alpha \in W \cap \Delta .
$$

Let $Q=\{\alpha \in \Delta ; \alpha(z) \geq 0\}$. Then $Q$ is a parabolic subset, so by (2.1), $Q=\Delta^{+} \bigsqcup M^{-}$. We have $\alpha \in M^{ \pm}$if and only if $\alpha(z)=0$, so by (2.3),

$$
W \cap \Delta=M^{+} \coprod M^{-} \text {. }
$$

By (2.2), (2.3) and (2.4),

$$
\mathfrak{m}=\mathfrak{h} \oplus \sum_{\left.\alpha\right|_{\mathfrak{z}}=0} \mathfrak{g}_{\alpha}=\mathfrak{h} \oplus \sum_{\alpha \in W \cap \Delta} \mathfrak{g}_{\alpha}=\mathfrak{h} \oplus \sum_{\alpha \in M^{ \pm}} \mathfrak{g}_{\alpha} .
$$

By comparing with (2.1), we see that $\mathfrak{m}$ is a Levi factor of the parabolic subalgebra $\mathfrak{h}+$ $\sum_{Q} \mathfrak{g}_{\alpha}$ 
Now we give the converse of Proposition 2.1.

Proposition 2.2 Let $\mathfrak{m}$ be a Levi factor. Then $\mathfrak{m}$ is the centralizer of its center.

Proof Since $\mathfrak{m}$ is a Levi factor, we can write $\mathfrak{m}=\mathfrak{h} \oplus \sum_{\alpha \in M^{ \pm}} \mathfrak{g}_{\alpha}$. Let $\Pi_{M}$ be the simple system for $\mathrm{M}^{+}$, and let

$$
\mathfrak{z}=\cap_{\alpha \in \Pi_{M}} \operatorname{ker}(\alpha) \subset \mathfrak{h} .
$$

Then $\mathfrak{z}$ is the center of $\mathfrak{m}$, and we want to show that $\mathfrak{m}$ is its centralizer, namely

$$
\mathfrak{m}=C(\mathfrak{z}) .
$$

The $\subset$ part of (2.5) is obvious, and it remains to prove the $\supset$ part. As before, choose $z \in \mathfrak{z}$ such that

$$
\begin{aligned}
& \text { (a) } \alpha(z)=0 \text { for all } \alpha \in \Pi_{M}, \\
& \text { (b) } \alpha(z)>0 \text { for all } \alpha \in \Pi \backslash \Pi_{M} \text {. }
\end{aligned}
$$

Suppose that $\mathfrak{g}_{\beta} \subset C(\mathfrak{z})$, where for $\beta=\sum_{\Pi} n_{\alpha} \alpha \in \Delta$. By (2.6)(a),

$$
0=\beta(z)=\sum_{\Pi} n_{\alpha} \alpha(z)=\sum_{\Pi \backslash \Pi_{M}} n_{\alpha} \alpha(z) .
$$

By (2.6)(b) and (2.7), $n_{\alpha}=0$ for all $\alpha \in \Pi \backslash \Pi_{M}$. Hence $\beta=\sum_{\Pi_{M}} n_{\alpha} \alpha$, which implies that $\mathfrak{g}_{\beta} \subset \mathfrak{m}$. This proves the $\supset$ part of (2.5).

Theorem 2.3 Let $\mathfrak{m}$ be a subalgebra of $\mathfrak{g}$, and let $\mathfrak{z}$ be the center of $\mathfrak{m}$. The following two conditions are equivalent.

(a) $\mathfrak{m}$ is the Levi factor of a parabolic subalgebra of $\mathfrak{g}$,

(b) $\mathfrak{z}$ is toral, and $\mathfrak{m}$ is the centralizer of $\mathfrak{z}$ in $\mathfrak{g}$.

Proof This follows from Propositions 2.1 and 2.2.

\section{Admissible Automorphisms}

In this section we prove Theorem 1.2. Let $\mathfrak{g}$ be a complex simple Lie algebra. Let $\mathfrak{h}$ be a Cartan subalgebra of $\mathfrak{g}$. Let $\Delta \subset \mathfrak{h}^{*}$ be the root system, and let $\mathfrak{g}=\mathfrak{h}+\sum_{\alpha \in \Delta} \mathfrak{g}_{\alpha}$ be the root space decomposition.

Let $\theta$ be an equal rank automorphism of $\mathfrak{g}$ of order $s$. By Theorem 1.1, we write $\mathfrak{g}^{\theta}=$ $\mathfrak{g}_{\mathrm{SS}}^{\theta}+\mathfrak{z}$, where $\mathfrak{g}_{\mathrm{sS}}^{\theta}$ and $\mathfrak{z}$ are respectively the semisimple part and the center. Define

$$
\Delta_{0}=\left\{\alpha \in \Delta ; \mathfrak{g}_{\alpha} \subset \mathfrak{g}_{\mathrm{ss}}^{\theta}\right\}, \quad \Delta_{1}=\left\{\alpha \in \Delta ; \mathfrak{g}_{\alpha} \not \subset \mathfrak{g}_{\mathrm{ss}}^{\theta}\right\} .
$$

If $\Delta^{+}$is a positive system of $\Delta$, we let $\Delta^{-}=-\Delta^{+}$as well as $\Delta_{0}^{ \pm}=\Delta_{0} \cap \Delta^{ \pm}$and $\Delta_{1}^{ \pm}=\Delta_{1} \cap \Delta^{ \pm}$. We give an equivalent formulation of the notion of admissible positive system defined in (1.4).

Definition 3.1 We say that $\Delta^{+}$is admissible for $\theta$ if

$$
\begin{aligned}
& \text { (a) }\left(\Delta_{0}+\Delta_{1}^{ \pm}\right) \cap \Delta \subset \Delta_{1}^{ \pm} \text {, } \\
& \text { (b) }\left(\Delta_{1}^{ \pm}+\Delta_{1}^{ \pm}\right) \cap \Delta_{0}=\emptyset .
\end{aligned}
$$


Note that the two notions of admissibility (1.4) and (3.1) coincide. In fact, since $\theta$ has order $s$, it leads to $\mathfrak{g}=\sum_{\mathbb{Z}_{s}} \mathfrak{g}_{m}$ of (1.3). Then $\mathfrak{g}^{\theta}=\mathfrak{h} \oplus \sum_{\alpha \in \Delta_{0}} \mathfrak{g}_{\alpha}$ and $\sum_{m \neq 0} \mathfrak{g}_{m}^{ \pm}=$ $\sum_{\alpha \in \Delta_{1}} \mathfrak{g}_{\alpha}$.

If a simple system $\Pi$ generates an admissible positive system for $\theta$, we also say that $\Pi$ is admissible.

Lemma 3.2 An admissible simple system for $\theta$ contains a simple system for $\Delta_{0}$.

Proof Let $\Pi$ be an admissible simple system for $\theta$. For $\alpha \in \Delta$, we recall its height ht $(\alpha)$ to be the sum of its coefficients as linear combination over $\Pi$. We prove by induction on the height of $\alpha \in \Delta_{0}^{+}$that $\alpha$ is a linear combination of members in $\Pi \cap \Delta_{0}$.

If $\operatorname{ht}(\alpha)=1$, then $\alpha \in \Pi$ and we are done. Suppose that $\operatorname{ht}(\alpha)>1$. Hence $\alpha \notin \Pi$ and we have

$$
\alpha=\beta+\gamma \in \Delta_{0}^{+},
$$

where $\beta, \gamma \in \Delta^{+}$. Then (3.1)(a) says that we cannot have $\beta \in \Delta_{0}^{+}$and $\gamma \in \Delta_{1}^{+}$, or vice versa. Also, (3.1)(b) says that we cannot have $\beta, \gamma \in \Delta_{1}^{+}$. Hence we must have $\beta, \gamma \in \Delta_{0}^{+}$. Since $\operatorname{ht}(\beta), \operatorname{ht}(\gamma)<\operatorname{ht}(\alpha)$, this completes the induction.

We have shown that if $\Pi=\left\{\alpha_{i}\right\} \cup\left\{\beta_{j}\right\}$ is an admissible simple system for $\theta$ with $\left\{\alpha_{i}\right\} \subset \Delta_{0}$ and $\left\{\beta_{j}\right\} \subset \Delta_{1}$, then every member of $\Delta_{0}^{+}$is a linear combination of $\left\{\alpha_{i}\right\}$. This implies that $\left\{\alpha_{i}\right\}$ is a simple system of $\Delta_{0}$.

Since $\theta$ is equal rank, we have that $\mathfrak{h} \subset \mathfrak{g}^{\theta}$ so that $\mathfrak{h}=\mathfrak{h}_{\mathrm{ss}} \oplus \mathfrak{z}$, and $\mathfrak{h}_{\mathrm{ss}}$ is a Cartan subalgebra of $\mathfrak{g}_{\mathrm{ss}}^{\theta}$. Since $\mathfrak{z}$ is the center of $\mathfrak{g}^{\theta}$, it acts trivially on $\mathfrak{g}_{\alpha} \subset \mathfrak{g}_{\mathrm{ss}}^{\theta}$, that is $\left.\alpha\right|_{\mathfrak{z}}=0$ for $\alpha \in \Delta_{0}$. Hence we may identify $\Delta_{0}$ with the root system of the semisimple Lie algebra $\mathfrak{g}_{\mathrm{ss}}^{\theta}$ by restricting $\alpha \in \Delta_{0}$ to $\mathfrak{h}_{\mathrm{ss}}$.

Proposition 3.3 Let $\theta$ be a finite order $\mathfrak{g}$-automorphism. If $\theta$ is admissible, then $\mathfrak{g}^{\theta}$ is a Levi factor.

Proof By Theorem 2.3, we need to show that $\mathfrak{g}^{\theta}$ is the centralizer of its center $\mathfrak{z}$, namely

$$
\mathfrak{g}^{\theta}=\mathfrak{h}+\sum_{\left.\alpha\right|_{\mathfrak{z}}=0} \mathfrak{g}_{\alpha}
$$

Suppose that $\theta$ is admissible. By definition, $\theta$ has an admissible positive system, and hence an admissible simple system $\Pi$ (see (1.4) and (3.1)). By Lemma 3.2, we have that

$$
\Pi=\left\{\alpha_{1}, \ldots \alpha_{r}, \alpha_{r+1}, \ldots, \alpha_{l}\right\}: \begin{aligned}
& \alpha_{1}, \ldots, \alpha_{r} \in \Delta_{1}, \\
& \\
& \alpha_{r+1}, \ldots, \alpha_{l} \in \Delta_{0} .
\end{aligned}
$$

Here $\alpha_{r+1}, \ldots, \alpha_{l}$ is a simple system for $\Delta_{0}$, and $r=\operatorname{dim}(\mathfrak{z})$, because $\operatorname{dim}(\mathfrak{h})=\operatorname{dim}\left(\mathfrak{h}_{\mathrm{ss}}\right)+$ $\operatorname{dim}(\mathfrak{z})$. Clearly $\left.\alpha_{i}\right|_{\mathfrak{z}}=0$ for $i=r+1, \ldots, l$, because such $\alpha_{i} \in \Delta_{0}$. Also, $\left.\alpha_{1}\right|_{\mathfrak{z}} \neq 0, \ldots$, $\left.\alpha_{r}\right|_{\mathfrak{z}} \neq 0$, for otherwise $\operatorname{dim}(\mathfrak{z})<r$.

Let $t \in \mathfrak{z}$ such that $\alpha_{i}(t)=1$ for all $i=1, \ldots, r$. Let $\alpha \in \Delta^{+}, \alpha=\sum_{i=1}^{r} n_{i} \alpha_{i}+$ $\sum_{j=r+1}^{l} m_{j} \alpha_{j}$. Then

$$
\alpha(t)=\sum_{i=1}^{r} n_{i} \alpha_{i}(t)+\sum_{j=r+1}^{l} m_{j} \alpha_{j}(t)=\sum_{i=1}^{r} n_{i} \geq 0
$$


So we have $\alpha \in \Delta_{1}^{+}$if and only if $\alpha(t)>0$, and $\alpha \in \Delta_{0}^{+}$if and only $\alpha(t)=0$. Hence

$$
\Delta_{0}=\left\{\alpha \in \Delta ;\left.\alpha\right|_{\mathfrak{z}}=0\right\}, \quad \Delta_{1}=\left\{\alpha \in \Delta ;\left.\alpha\right|_{\mathfrak{z}} \neq 0\right\} .
$$

This concludes our proof.

Proposition 3.4 Let $\mathfrak{m} \subset \mathfrak{g}$ be a Levi factor. There exists a finite order $\mathfrak{g}$-automorphism $\theta$ such that $\mathfrak{g}^{\theta}=\mathfrak{m}$.

Proof Let $\mathfrak{m}$ be a Levi factor. There exists a parabolic subalgebra $\mathfrak{q}$ of $\mathfrak{g}$ such that $\mathfrak{q}=\mathfrak{m}+\mathfrak{n}$, where $\mathfrak{n}$ is a nilpotent ideal of $\mathfrak{q}$. Let $\Delta^{+}$be a positive system as given in (2.1). Let $M^{+}$ denote the positive roots of $\mathfrak{m}$. The extended Dynkin diagram $\mathrm{D}$ represents $\mathrm{D}=\Pi \cup\{\varphi\}$, where $\varphi$ is the lowest root. By (2.1), we have a partition $\Pi=\Pi_{M} \cup \Pi_{N}$, where $\Pi_{M}=$ $\Pi \cap M^{+}$is a simple system of $\mathfrak{m}$, and $\Pi_{N}$ are simple roots which belong to $\mathfrak{n}$.

Let $k$ be the Kac diagram given by

$$
k_{\alpha}=\left\{\begin{array}{l}
0 \text { for } \alpha \in \Pi_{M}, \\
1 \text { for } \alpha \in \Pi_{N} \cup\{\varphi\} .
\end{array}\right.
$$

By Theorem 1.1, it represents a finite order $\mathfrak{g}$-representation $\theta$ such that the subdiagram ker $k=\left\{\alpha \in \mathrm{D} ; k_{\alpha}=0\right\}$ is the Dynkin diagram of $\mathfrak{g}_{\mathrm{ss}}^{\theta}$. By (3.2), ker $k=\Pi_{M}$, so $\mathfrak{g}_{\mathrm{ss}}^{\theta}=\mathfrak{m}_{\mathrm{ss}}$. The centers of $\mathfrak{g}^{\theta}$ and $\mathfrak{m}$ both consist of members of $\mathfrak{h}$ which are annihilated by $\Pi_{M}$, so they coincide. Hence $\mathfrak{g}^{\theta}=\mathfrak{m}$.

Proposition 3.5 Let $\theta$ be a finite order $\mathfrak{g}$-automorphism. If $\mathfrak{g}^{\theta}$ is a Levi factor, then $\theta$ is admissible.

Proof Write $\mathfrak{g}^{\theta}=\mathfrak{g}_{\mathrm{sS}}^{\theta}+\mathfrak{z}$ as before, and suppose that $\mathfrak{g}^{\theta}$ is a Levi factor. By (2.1), there exists a simple system $\Pi \supset \Pi_{M}$ for $\Delta$, with $\Pi_{M}$ a simple system for $\Delta_{0}$, the root system of $\mathfrak{g}_{\mathrm{ss}}^{\theta}$. By Proposition 2.2, there exists a $z \in \mathfrak{z}$ such that

$$
\alpha(z)\left\{\begin{array}{l}
=0 \text { for } \alpha \in \Pi_{M} \\
>0 \text { for } \alpha \in \Pi \backslash \Pi_{M} .
\end{array}\right.
$$

Hence, with respect to the positive system given by $\Pi$, we can characterize $\Delta_{0}$ and $\Delta_{1}$ as

$$
\Delta_{0}=\{\alpha \in \Delta \mid \alpha(z)=0\}, \quad \Delta_{1}^{+}=\{\alpha \in \Delta \mid \alpha(z)>0\}, \quad \Delta_{1}^{-}=\{\alpha \in \Delta \mid \alpha(z)<0\} .
$$

It leads to (3.1), which defines an admissible system for $\theta$.

Proof of Theorem 1.2 Let $\theta$ be a finite order equal rank admissible $\mathfrak{g}$-automorphism. By Proposition 3.3, $\mathfrak{g}^{\theta}$ is a Levi factor of $\mathfrak{g}$. Conversely, let $\mathfrak{m}$ be a Levi factor of $\mathfrak{g}$. By Proposition 3.4, there exists a finite order $\mathfrak{g}$-automorphism $\theta$ such that $\mathfrak{g}^{\theta}=\mathfrak{m}$. By Proposition 3.5, $\theta$ is admissible. This proves Theorem 1.2.

\section{Kac Diagrams}

In this section, we prove Theorem 1.3. Let $\theta$ be an equal rank finite order $\mathfrak{g}$-automorphism, and $\mathfrak{g}^{\theta}=\mathfrak{g}_{\mathrm{sS}}^{\theta}+\mathfrak{z}$. Let $\mathrm{D}$ be the extended Dynkin diagram, and let $k$ be the Kac diagram of $\theta$. Let

$$
\operatorname{ker} k=\left\{\alpha \in \mathrm{D} ; k_{\alpha}=0\right\} \text {. }
$$


The labels $\left\{a_{\alpha}\right\}_{\mathrm{D}}$ satisfy $\sum_{\mathrm{D}} a_{\alpha} \alpha=0$, and $k$ is said to be relatively prime if $\left\{a_{\alpha}\right\}_{\mathrm{D} \backslash \text { ker } k}$ are relatively prime. Let $C(\mathfrak{z})$ be the centralizer of $\mathfrak{z}$ in $\mathfrak{g}$.

Proposition 4.1 If $k$ is relatively prime, then $C(\mathfrak{z})=\mathfrak{g}^{\theta}$.

Proof Since $\mathfrak{z}$ is the center of $\mathfrak{g}^{\theta}$, it is obvious that $\mathfrak{g}^{\theta} \subset C(\mathfrak{z})$. So we only have to show that $C(\mathfrak{z}) \subset \mathfrak{g}^{\theta}$. Since both $C(\mathfrak{z})$ and $\mathfrak{g}^{\theta}$ are sums of the Cartan subalgebra and some root spaces, it suffices to show that if $\mathfrak{g}_{\beta} \subset C(\mathfrak{z})$, then $\mathfrak{g}_{\beta} \subset \mathfrak{g}^{\theta}$.

Let $\mathfrak{g}_{\beta} \subset C(\mathfrak{z})$. Then $\beta$ annihilates $\mathfrak{z}$. Write

$$
\beta=\sum_{\mathrm{D}} r_{\alpha} \alpha
$$

This expression is not unique because $\mathrm{D}$ is linearly dependent, but this is not a problem. Here ker $k$ are roots of $\mathfrak{g}^{\theta}$. If $\left\{r_{\alpha}\right\}_{\mathrm{D} \backslash \text { ker } k}$ are all 0 , then $\beta=\sum_{\operatorname{ker} k} r_{\alpha} \alpha$ is a root of $\mathfrak{g}^{\theta}$ and there is nothing to prove. So we may assume that

$$
r_{\delta} \neq 0 \text { for some } \delta \in \mathrm{D} \backslash \operatorname{ker} k .
$$

Since $\mathfrak{z}$ is annihilated by ker $k$, we have

$$
0=\beta(\mathfrak{z})=\sum_{\mathrm{D}} r_{\alpha} \alpha(\mathfrak{z})=\sum_{\mathrm{D} \backslash \operatorname{ker} k} r_{\alpha} \alpha(\mathfrak{z}) .
$$

We also have

$$
0=\sum_{\mathrm{D}} a_{\alpha} \alpha(\mathfrak{z})=\sum_{\mathrm{D} \backslash \text { ker } k} a_{\alpha} \alpha(\mathfrak{z})
$$

Let $\mathbb{C}(\mathrm{D} \backslash \operatorname{ker} k)$ be the linear span of $\mathrm{D} \backslash$ ker $k$. Consider the linear map

$$
\phi: \mathbb{C}(\mathrm{D} \backslash \operatorname{ker} k) \longrightarrow \mathfrak{z}^{*},
$$

where $\phi(\alpha)$ is the restriction of $\alpha$ to $\mathfrak{z}$. It is surjective. By Theorem $1.1, \operatorname{dim} \mathfrak{z}=$ $\sharp(\mathrm{D} \backslash \operatorname{ker} k)-1, \operatorname{sod} \operatorname{dim}(\operatorname{ker} \phi)=1$. By (4.3) and (4.4), $\sum_{\mathrm{D} \backslash \operatorname{ker} k} r_{\alpha} \alpha, \sum_{\mathrm{D} \backslash \operatorname{ker} k} a_{\alpha} \alpha \in \operatorname{ker} \phi$, so they are linearly dependent. By (4.2), $\sum_{\mathrm{D} \backslash \text { ker } k} r_{\alpha} \alpha \neq 0$, so there exists $t \in \mathbb{C}$ such that

$$
t r_{\alpha}=a_{\alpha} \text { for all } \alpha \in \mathrm{D} \backslash \operatorname{ker} k .
$$

In particular $t \in \mathbb{Z}$ because $\alpha$ is a root. But $\left\{a_{\alpha}\right\}_{\mathrm{D}}$ are relatively prime, so $t= \pm 1$. We assume that $t=1$ (similar arguments for $t=-1$ ), so (4.1) becomes

$$
\beta=\sum_{\text {ker } k} r_{\alpha} \alpha+\sum_{\mathrm{D} \backslash \operatorname{ker} k} a_{\alpha} \alpha
$$

Let $s=\sum_{\mathrm{D} \backslash \text { ker } k} a_{\alpha} k_{\alpha}$, and let $\omega=\exp (2 \pi i / s)$. Then $\theta$ has order $s$, and

$$
\left.\theta\right|_{\mathfrak{g}_{\alpha}}=\left\{\begin{array}{cl}
\omega^{k_{\alpha}} & \text { for } \alpha \in \mathrm{D} \backslash \operatorname{ker} k, \\
1 & \text { for } \alpha \in \operatorname{ker} k .
\end{array}\right.
$$

By (4.5), $\theta$ has eigenvalue $\omega^{s}=1$ on $\mathfrak{g}_{\beta}$, namely $\mathfrak{g}_{\beta} \subset \mathfrak{g}^{\theta}$. This proves that $C(\mathfrak{z}) \subset \mathfrak{g}^{\theta}$, and the proposition follows.

The converse of Proposition 4.1 is given below.

Proposition 4.2 Let $k$ be the Kac diagram of a $\mathfrak{g}$-automorphism $\theta$. If $k$ is not relatively prime, then $C(\mathfrak{z}) \not \subset \mathfrak{g}^{\theta}$. 
Proof Suppose that a Kac diagram $k$ is not relatively prime, namely there exist positive integers $t \neq 1$ and $\left\{r_{\alpha}\right\}_{\mathrm{D} \backslash \text { ker } k}$ such that

$$
a_{\alpha}=t r_{\alpha} \text { for all } \alpha \in \mathrm{D} \backslash \operatorname{ker} k .
$$

Then there exist integers $\left\{r_{\alpha}\right\}_{\text {ker } k}$ such that

$$
\beta=\sum_{\mathrm{D}} r_{\alpha} \alpha
$$

is a root; see Remark 4.3 below. Let $s=\sum_{\mathrm{D} \backslash \text { ker } k} a_{\alpha} k_{\alpha}$, and let $\omega=\exp (2 \pi i / s)$. Then $\theta$ has order $s$ and satisfies (4.6). Since $\omega^{\sum_{\mathrm{D}} r_{\alpha} k_{\alpha}} \neq 1$, it follows that

$$
\mathfrak{g}_{\beta} \not \subset \mathfrak{g}^{\theta} \text {. }
$$

Let $\mathfrak{z}$ be the center of $\mathfrak{g}^{\theta}$. Similar to (4.4), we have $\sum_{\mathrm{D} \backslash \operatorname{ker} k} a_{\alpha} \alpha(\mathfrak{z})=0$. So by (4.7), $\sum_{\mathrm{D} \backslash \text { kerk }} r_{\alpha} \alpha(\mathfrak{z})=0$. It implies that

$$
\beta(\mathfrak{z})=\sum_{\mathrm{D}} r_{\alpha} \alpha(\mathfrak{z})=0,
$$

and hence $\mathfrak{g}_{\beta} \subset C(\mathfrak{z})$. This, together with (4.9), imply that $C(\mathfrak{z}) \not \subset \mathfrak{g}^{\theta}$.

Remark 4.3 Construction of the root $\beta$ in (4.8). Let $k$ be a Kac diagram. Suppose that $x, y \in \mathrm{D} \backslash \operatorname{ker} k$, and $a_{x}, a_{y}$ have a nontrivial common factor $t$ in (4.7).

If $\mathfrak{g}$ is not one of $E_{7}, E_{8}, F_{4}$, then the list of extended Dynkin diagrams shows that $a_{x}=a_{y} \in\{2,3\}$, so $r_{x}=r_{y}=1$ in (4.7). We may let $S \subset \mathrm{D}$ be the smallest connected subdiagram containing $\mathrm{D} \backslash \operatorname{ker} k$, then $\beta=\sum_{S} \alpha$ satisfies (4.8).

If $\mathfrak{g}$ is one of $E_{7}, E_{8}, F_{4}$, then $\left\{a_{x}, a_{y}\right\}$ can possibly be $\{2,4\}$ or $\{3,6\}$, so that $\left\{r_{x}, r_{y}\right\}=$ $\{1,2\}$ in (4.7). Nevertheless, the list of roots (see for instance [8, Appendix C]) shows that a root $\beta$ of (4.8) exists. For example in $E_{7}$, suppose that $\{x, y\}=\mathrm{D} \backslash \operatorname{ker} k$ with $a_{x}=4=2 r_{x}$ and $a_{y}=2=2 r_{y}$. Let $\beta$ be the positive root whose coefficients on the Dynkin diagram are $\left(\begin{array}{cccccc}1 & 1 & 2 & 2 & 1 & 1\end{array}\right)$. Then it can be expressed as $\beta=2 x+y+\sum_{\mathrm{ker} k} r_{\alpha} \alpha$ of (4.8).

Proof of Theorem 1.3 Let $\theta$ be an equal rank finite order $\mathfrak{g}$-automorphism with Kac diagram $k$, and let $\mathfrak{z}$ be the center of $\mathfrak{g}^{\theta}$. We have

$$
\begin{array}{rlr}
\left\{a_{\alpha}\right\}_{\mathrm{D} \backslash \text { ker } k \text { relatively prime }} & \Longleftrightarrow C(\mathfrak{g})=\mathfrak{g}^{\theta} \quad \text { by Propositions } 4.1 \text { and } 4.2 \\
& \Longleftrightarrow \mathfrak{g}^{\theta} \text { is Levi } & \text { by Theorem } 2.3 \\
& \Longleftrightarrow \theta \text { is admissible. } & \text { by Theorem } 1.2
\end{array}
$$

This proves the theorem.

\section{Automorphisms of Minimal Orders}

In this section, we prove Theorem 1.4. Let $\theta$ be an equal rank finite order $\mathfrak{g}$-automorphism. By Theorem 1.1, it is represented by a Kac diagram $k$. A circling on D is a choice to circle some of the vertices of $\mathrm{D}$. The Kac diagram $k$ leads to a circling $c$ as follows: For all $\alpha \in \mathrm{D}$,

$$
\alpha\left\{\begin{array}{l}
\text { not circled if } k_{\alpha}=0 \\
\text { circled if } k_{\alpha}>0
\end{array}\right.
$$


Let $c(\mathrm{D})$ denote the circled vertices. More generally for $X \subset \mathrm{D}$,

$$
c(X)=\text { circled vertices of } X \text {. }
$$

We say that $c$ represents a reductive subalgebra $\mathfrak{m}=\mathfrak{m}_{\mathrm{ss}}+\mathfrak{z}$ of $\mathfrak{g}$ (where $\mathfrak{m}_{\mathrm{ss}}$ is semisimple and $\mathfrak{z}$ is the center) if

$$
\operatorname{Dyn}\left(\mathfrak{m}_{\mathrm{ss}}\right)=\mathrm{D} \backslash c(\mathrm{D}), \operatorname{dim} \mathfrak{z}=|c(\mathrm{D})|-1 .
$$

So if $k$ represents $\theta$, then by (5.1), $c$ represents $\mathfrak{g}^{\theta}$. By Theorems 1.1, 1.2 and 1.3, $\mathfrak{g}^{\theta}$ is a Levi factor if and only if $\left\{a_{\alpha}\right\}_{c(\mathrm{D})}$ are relatively prime, where $\left\{a_{\alpha}\right\}_{\mathrm{D}}$ are the labels $\sum_{\mathrm{D}} a_{\alpha} \alpha=0$. The vertices of $D$ represent any set of roots of the form

$$
\widetilde{\Pi}=\Pi \cup\{\varphi\},
$$

where $\Pi$ is a simple system with lowest root $\varphi$.

Since $\theta$ is equal rank, it acts trivially on a Cartan subalgebra of $\mathfrak{g}$ and stabilizes its root spaces $\mathfrak{g}_{\alpha}$. We can define a circling $c^{\prime}$ on D (with respect to some $\widetilde{\Pi}$ ) by

$$
c^{\prime}(\mathrm{D})=\left\{\alpha \in \mathrm{D} ;\left.\theta\right|_{\mathfrak{g}_{\alpha}} \neq 1\right\} .
$$

While $\theta$ determines $c$ uniquely up to diagram automorphism by (5.1), it leads to several $c^{\prime}$ by (5.5) due to various choices of $\widetilde{\Pi}$.

Lemma 5.1 Fix an equal rank finite order $\mathfrak{g}$-automorphism $\theta$. Let $c$ be obtained by (5.1), and let $c^{\prime}$ be obtained by (5.5). Then $|c(\mathrm{D})| \leq\left|c^{\prime}(\mathrm{D})\right|$.

Proof By Theorem 1.1, $\mathfrak{g}^{\theta}$ is reductive, and its semisimple part $\mathfrak{g}_{\mathrm{ss}}^{\theta}$ has Dynkin diagram formed by $\left\{\alpha \in \mathrm{D} ; k_{\alpha}=0\right\}$. Namely,

$$
|\mathrm{D} \backslash c(\mathrm{D})|=\operatorname{rank}\left(\mathfrak{g}_{\mathrm{ss}}^{\theta}\right) .
$$

Now let the vertices of D represent some $\widetilde{\Pi}=\Pi \cup\{\varphi\}$ as discussed in (5.4). For each $\alpha \in \widetilde{\Pi}$, if $\alpha$ is not circled by $c^{\prime}$, then $\mathfrak{g}_{\alpha} \subset \mathfrak{g}_{\mathrm{ss}}^{\theta}$. So $\widetilde{\Pi} \backslash c^{\prime}(\widetilde{\Pi})$ are linearly independent roots of $\mathfrak{g}_{\mathrm{ss}}^{\theta}$, hence

$$
\left|\mathrm{D} \backslash c^{\prime}(\mathrm{D})\right| \leq \operatorname{rank}\left(\mathfrak{g}_{\mathrm{ss}}^{\theta}\right) .
$$

The lemma follows from (5.6) and (5.7).

We illustrate the arguments of Lemma 5.1 with an example. Let $\mathfrak{g}=\mathfrak{s l}(4, \mathbb{C})$, with extended Dynkin diagram D in Fig. 1. Consider the Kac diagram $k$ in Fig. 1a. It represents an automorphism $\theta$ of order 3. By (5.1), it leads to a circling $c$ given by Fig. 1b. We choose the simple system such that $c$ (D) consists of the simple roots, and the lowest root is not circled. Let the Cartan subalgebra be the diagonal matrices, and let $\epsilon_{i}-\epsilon_{j}$ be its roots in

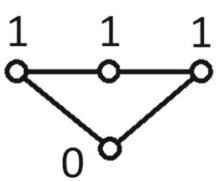

(a)

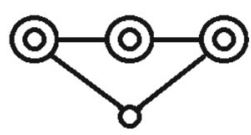

(b)

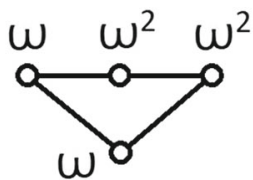

(c)

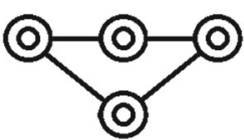

(d)

Fig. 1 Comparison on numbers of circled vertices 
the usual way such that the simple roots are $\left\{\epsilon_{i}-\epsilon_{i+1}\right\}_{1}^{3}$. Let $\omega=\exp (2 \pi i / 3)$. Then the eigenvalues of $\theta$ on the root spaces are

$$
\left(\begin{array}{cccc}
* & \omega & \omega^{2} & 1 \\
\omega^{2} & * & \omega & \omega^{2} \\
\omega & \omega^{2} & * & \omega \\
1 & \omega & \omega^{2} & *
\end{array}\right) .
$$

For example, $\theta$ has eigenvalue $\omega^{2}$ on $E_{13}$ because

$$
\theta E_{13}=\theta\left[E_{12}, E_{23}\right]=\left[\theta E_{12}, \theta E_{23}\right]=\left[\omega E_{12}, \omega E_{23}\right]=\omega^{2} E_{13} .
$$

Consider another simple system $\Pi=\left\{\epsilon_{1}-\epsilon_{2}, \epsilon_{2}-\epsilon_{4}, \epsilon_{4}-\epsilon_{3}\right\}$, with lowest root $\varphi=$ $\epsilon_{3}-\epsilon_{1}$. If we now let $D$ represent $\Pi \cup\{\varphi\}$, then by (5.8), the eigenvalues of $\theta$ on their root spaces are given by Fig. 1c. By (5.5), the resulting circling $c^{\prime}$ is Fig. 1d. We have $|c(\mathrm{D})|=3<4=\left|c^{\prime}(\mathrm{D})\right|$, which is consistent with Lemma 5.1. We also see from (5.8) that only the root spaces of $\pm\left(\epsilon_{1}-\epsilon_{4}\right)$ have eigenvalue 1 , so (5.5) cannot lead to a circling with fewer than 3 circled vertices.

Two Levi factors $\mathfrak{m}, \mathfrak{m}^{\prime} \subset \mathfrak{g}$ are said to be isomorphic if there is an isomorphism $\sigma$ : $\mathfrak{m} \longrightarrow \mathfrak{m}^{\prime}$. If in addition $\sigma$ is the restriction of an automorphism $\mathfrak{g} \longrightarrow \mathfrak{g}$, we say that $\mathfrak{m}, \mathfrak{m}^{\prime}$ are strongly isomorphic. We write

$$
\mathfrak{m} \simeq \mathfrak{m}^{\prime}, \mathfrak{m} \cong \mathfrak{m}^{\prime}
$$

for isomorphism and strong isomorphism respectively.

Obviously if $\mathfrak{m}, \mathfrak{m}^{\prime}$ have different root lengths, they cannot be strongly isomorphic. So in Fig. 2a,b, we have $\mathfrak{m} \simeq \mathfrak{m}^{\prime} \simeq A_{1}+\mathbb{C}$ by (5.3), but they are not strongly isomorphic. However, even with the same root lengths, they may not be strongly isomorphic. Consider Fig. 2c,d, where by (5.3), $\mathfrak{m} \simeq \mathfrak{m}^{\prime} \simeq A_{1}^{2}+\mathbb{C}^{4}$ have centers $\mathfrak{z} \simeq \mathfrak{z}^{\prime} \simeq \mathbb{C}^{4}$. If $\mathfrak{m}, \mathfrak{m}^{\prime}$ were strongly isomorphic, the decomposition in [9, Sec. 1] would yield isomorphic Kostant root systems $R \cong R^{\prime}$. This is because $\mathfrak{z}, \mathfrak{z}^{\prime}$ would be conjugate, and $R, R^{\prime}$ are obtained from the decompositions of $\mathfrak{g}$ under the adjoint actions of $\mathfrak{z}, \mathfrak{z}^{\prime}$. We check that, with the notation of [9, Sec. 2.3],

$$
\begin{aligned}
R^{+}= & \left\{\mu_{i}\right\}_{1}^{4} \cup\left\{\mu_{1}+\mu_{2}, \mu_{2}+\mu_{3}, \mu_{2}+\mu_{4}, \mu_{2}+\mu_{3}+\mu_{4},\right. \\
& \mu_{1}+\mu_{2}+\mu_{3}, \mu_{1}+\mu_{2}+\mu_{4}, \mu_{1}+\mu_{2}+\mu_{3}+\mu_{4}, 2 \mu_{2}+\mu_{3}+\mu_{4}, \\
& \left.\mu_{1}+2 \mu_{2}+\mu_{3}+\mu_{4}, 2 \mu_{1}+2 \mu_{2}+\mu_{3}+\mu_{4}\right\}, \\
\left(R^{\prime}\right)^{+}= & \left\{\mu_{i}\right\}_{1}^{4} \cup\left\{\mu_{i}+\mu_{i+1}\right\}_{1}^{3} \cup\left\{\mu_{i}+\mu_{i+1}+\mu_{i+2}\right\}_{1}^{2} \\
& \cup\left\{\mu_{1}+\mu_{2}+\mu_{3}+\mu_{4}, \mu_{3}+2 \mu_{4}, \mu_{2}+\mu_{3}+2 \mu_{4}, \mu_{1}+\mu_{2}+\mu_{3}+2 \mu_{4},\right. \\
& \left.\mu_{2}+2 \mu_{3}+2 \mu_{4}, \mu_{1}+\mu_{2}+2 \mu_{3}+2 \mu_{4}, \mu_{1}+2 \mu_{2}+2 \mu_{3}+2 \mu_{4}\right\} .
\end{aligned}
$$

So $\left|R^{+}\right|=14$ and $\left|\left(R^{\prime}\right)^{+}\right|=16$, and hence $R \approx R^{\prime}$. Therefore, $\mathfrak{m}$ and $\mathfrak{m}^{\prime}$ are not strongly isomorphic in Fig. 2c,d.

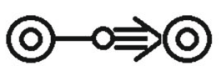

(a)

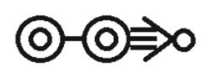

(b)

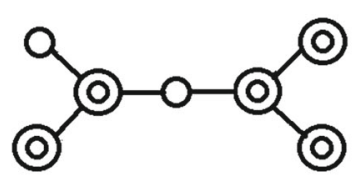

(c)

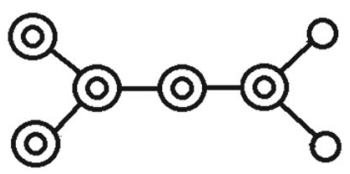

(d)

Fig. 2 Some circlings on $G_{2}$ and $D_{6}$ 
We make this convention from here through Proposition 5.3: When we write a simple system of $A_{n}$ as $\left\{\alpha_{1}, \ldots, \alpha_{n}\right\}$, each $\alpha_{i}$ is not orthogonal to $\alpha_{i+1}$, namely they are adjacent in the Dynkin diagram.

We describe a combinatorial method which shows strong isomorphism $\mathfrak{m} \cong \mathfrak{m}^{\prime}$. Given a circling $c$, we define a slidable region to be a connected subdiagram $R \subset \mathrm{D}$ of type $A$ such that:

(a) exactly one vertex of $R$ is circled,

(b) if $\alpha \in \mathrm{D} \backslash R$ is adjacent to $R$, then $\alpha$ is circled.

We define a new circling $c^{\prime}$ (with respect to a slidable region $R$ ) by

(a) $\left.c\right|_{R},\left.c^{\prime}\right|_{R}$ are related by diagram symmetry of the subdiagram $R$,

(b) $c, c^{\prime}$ coincide on $\mathrm{D} \backslash R$.

The process $c \rightsquigarrow c^{\prime}$ is called sliding because (5.10)(a) says that the circled vertex of $R \cong A_{n}$ appears to slide from location $i$ to location $n+1-i$. For example, Fig. 3a has two slidable regions as indicated. Their slidings are shown by the arrows, and one of them leads to Fig. 3b. We say that two circlings are related by sliding if one leads to the other by several steps of sliding.

We shall prove Proposition 5.3, which says that sliding is an effective method to check strong isomorphism. For this purpose, we first prove the following. Let $W$ be the Weyl group of $A_{n-1}$.

Lemma 5.2 Let $\Pi=\left\{\alpha_{1}, \ldots, \alpha_{n-1}\right\}$ be a simple system of $A_{n-1}(n>1)$. Fix an integer $1 \leq p<n-p$. Then there exists $w \in W$ such that

$$
w\left(\alpha_{i}\right)=\left\{\begin{array}{cl}
\alpha_{i+p} & 1 \leq i<n-p \\
-\left(\alpha_{1}+\ldots+\alpha_{n-1}\right) & i=n-p \\
\alpha_{i-(n-p)} & n-p<i \leq n-1
\end{array}\right.
$$

Proof We express the roots of $A_{n-1}$ as $\epsilon_{i}-\epsilon_{j}$ in the usual way. There is a bijective correspondence between $W$ and the symmetric group $S_{n}$, where $w \in W$ is associated to $\tau \in S_{n}$ by

$$
w\left(\epsilon_{i}-\epsilon_{j}\right)=\epsilon_{\tau(i)}-\epsilon_{\tau(j)}
$$

Let $w$ be associated to $\tau$ given by

$$
\tau(i)=\left\{\begin{array}{cc}
i+p & 1 \leq i \leq n-p \\
i-(n-p) & n-p<i \leq n .
\end{array}\right.
$$

Let $\alpha_{i}=\epsilon_{i}-\epsilon_{i+1}$. By (5.12) and (5.13),

$$
\begin{aligned}
& 1 \leq i<n-p: w\left(\alpha_{i}\right)=w\left(\epsilon_{i}-\epsilon_{i+1}\right)=\epsilon_{p+i}-\epsilon_{p+i+1}=\alpha_{i+p}, \\
& w\left(\alpha_{n-p}\right)=w\left(\epsilon_{n-p}-\epsilon_{n-p+1}\right)=\epsilon_{n}-\epsilon_{1}=-\left(\alpha_{1}+\cdots+\alpha_{n-1}\right), \\
& n-p<i \leq n-1: w\left(\alpha_{i}\right)=w\left(\epsilon_{i}-\epsilon_{i+1}\right)=\epsilon_{i-(n-p)}-\epsilon_{i+1-(n-p)}=\alpha_{i-(n-p)} .
\end{aligned}
$$

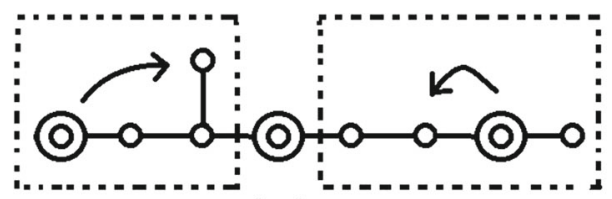

(a)

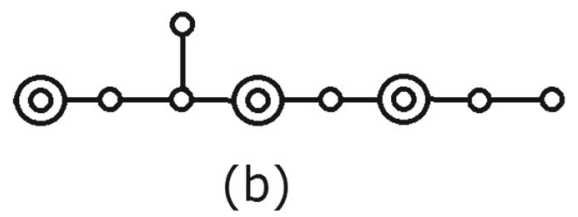

Fig. 3 Sliding 
Hence $w$ satisfies (5.11), and the lemma follows.

Proposition 5.3 Let $\mathfrak{m}$ and $\mathfrak{m}^{\prime}$ be Levi factors, represented by circlings $c$ and $c^{\prime}$ on $\mathrm{D}$ by (5.3). If $c$ and $c^{\prime}$ are related by sliding, then $\mathfrak{m} \cong \mathfrak{m}^{\prime}$.

Proof Let $\mathfrak{m}$ be a Levi factor, and let $c$ be the corresponding circling on D by (5.3). Recall from (5.4) that $\widetilde{\Pi}$ denotes a simple system with its lowest root, and the vertices of D represent $\widetilde{\Pi}$. Let $k$ be the Kac diagram given by

$$
k_{\alpha}=\left\{\begin{array}{l}
1 \text { if } \alpha \in c(\mathrm{D}), \\
0 \text { if } \alpha \in \mathrm{D} \backslash c(\mathrm{D}) .
\end{array}\right.
$$

Here $c$ and $k$ satisfy (5.1). Recall that $\left\{a_{\alpha}\right\}_{\mathrm{D}}$ are the canonical positive integers which satisfy $\sum_{\mathrm{D}} a_{\alpha} \alpha=0$. By Theorem $1.1, k$ represents a $\mathfrak{g}$-automorphism $\theta$ of order $N=\sum_{c(\mathrm{D})} a_{\alpha}$, and its invariant subalgebra is $\mathfrak{g}^{\theta}=\mathfrak{m}$.

Let $R$ be a slidable region of $c$. It is of type $A$, so we write $R=\left\{\alpha_{1}, \ldots, \alpha_{n-1}\right\}$, and

$$
c(R)=\left\{\alpha_{p}\right\}
$$

for some $1 \leq p \leq n-1$. Here $c(R)$ denotes the vertices of $R$ that are circled, see (5.2). Let $\omega=\exp (2 \pi i / N)$. The eigenvalues of $\theta$ satisfy

$$
\left.\theta\right|_{\mathfrak{g}_{\alpha_{p}}}=\omega,\left.\quad \theta\right|_{\mathfrak{g}_{\alpha_{i}}}=1 \text { for all } i \neq p .
$$

Reverse the numbering if necessary, we may assume that $p$ is small enough so that $p<$ $n-p$.

The slidable region $R$ is the basis of a closed subroot system $\Delta_{R} \subset \Delta$ of type $A_{n-1}$, and we let $\mathfrak{g}_{R}$ be the simple subalgebra of $\mathfrak{g}$ whose root system is $\Delta_{R}$. Then $R$ is the Dynkin diagram of $\mathfrak{g}_{R}$. Let $W_{R} \subset$ aut $\left(\Delta_{R}\right)$ be the Weyl group of $\Delta_{R}$.

Let $w \in W_{R}$ be given by Lemma 5.2. It is a product of reflections $r_{\alpha}$ where $\alpha \in \Delta_{R}$. By regarding $\alpha$ as a member of $\Delta$, each $r_{\alpha}$ extends naturally to aut $(\Delta)$, so $w$ also extends to some $f \in \operatorname{aut}(\Delta)$. By Lemma 5.2, $f$ satisfies (5.11).

Suppose that we now let D represent $f(\widetilde{\Pi})$ instead. By (5.11) and (5.15), for all $\alpha \in$ $f(\widetilde{\Pi})=\mathrm{D}$,

$$
\left.\theta\right|_{\mathfrak{g}_{\alpha}}=\left\{\begin{array}{cl}
\omega^{-1} & \text { if } \alpha=f\left(\alpha_{n-p}\right) \\
1 & \text { if } \alpha=f\left(\alpha_{i}\right), i \in\{1, \ldots, n-1\} \backslash\{n-p\} \\
\omega^{k_{\alpha}} & \text { if } \alpha \in \mathrm{D} \backslash\left\{f\left(\alpha_{i}\right)\right\}_{1}^{n-1} .
\end{array}\right.
$$

Let $c^{\prime}$ be obtained by (5.5), namely it circles $\alpha \in f(\widetilde{\Pi})$ if and only if $\left.\theta\right|_{\mathfrak{g}_{\alpha}} \neq 1$. By (5.14) and (5.16),

$$
c(R)=\left\{\alpha_{p}\right\}, c^{\prime}(R)=\left\{f\left(\alpha_{n-p}\right)\right\} .
$$

Since $c$ and $c^{\prime}$ are circlings with respect to $\widetilde{\Pi}$ and $f(\widetilde{\Pi})$ respectively, (5.17) says that $\left.c\right|_{R}$ and $\left.c^{\prime}\right|_{R}$ are related by the diagram symmetry of $R$.

Let $\partial R$ denote the vertices of $\mathrm{D} \backslash R$ that are adjacent to $R$. Since $R$ is a sliding region with respect to $c$, by (5.9)(b),

$$
c(\partial R)=\partial R .
$$

When we extend $w \in W_{R}$ to $f \in \operatorname{aut}(\Delta)$, the roots that are orthogonal to $R$ remain fixed by $f$, hence

By (5.17) and (5.18),

$$
c(\mathrm{D} \backslash(R \cup \partial R))=c^{\prime}(\mathrm{D} \backslash(R \cup \partial R)) .
$$

$$
\begin{aligned}
|c(\mathrm{D})| & =|c(R)|+|c(\partial R)|+|c(\mathrm{D} \backslash(R \cup \partial R))| \\
& =1+|\partial R|+|c(\mathrm{D} \backslash(R \cup \partial R))| .
\end{aligned}
$$


By (5.17) and (5.19)

$$
\begin{aligned}
\left|c^{\prime}(\mathrm{D})\right| & =\left|c^{\prime}(R)\right|+\left|c^{\prime}(\partial R)\right|+\left|c^{\prime}(\mathrm{D} \backslash(R \cup \partial R))\right| \\
& =1+\left|c^{\prime}(\partial R)\right|+|c(\mathrm{D} \backslash(R \cup \partial R))| .
\end{aligned}
$$

By Lemma 5.1, $|c(\mathrm{D})| \leq\left|c^{\prime}(\mathrm{D})\right|$. So (5.20) and (5.21) imply that $c^{\prime}(\partial R)=\partial R$. We conclude that $c$ and $c^{\prime}$ are related by sliding on $R$.

Since $c$ and $c^{\prime}$ are related by sliding, the subdiagrams $\mathrm{D} \backslash c(\mathrm{D})$ and $\mathrm{D} \backslash c^{\prime}(\mathrm{D})$ are isomorphic, namely

$$
\operatorname{Dyn}\left(\mathfrak{m}_{\mathrm{ss}}\right)=\mathrm{D} \backslash c^{\prime}(\mathrm{D}) \text { with respect to } f(\widetilde{\Pi}) .
$$

By the hypothesis of this proposition, suppose that

$$
\operatorname{Dyn}\left(\mathfrak{m}_{\mathrm{ss}}^{\prime}\right)=\mathrm{D} \backslash c^{\prime}(\mathrm{D}) \text { with respect to some } \widetilde{\Pi}^{\prime} .
$$

Let $h$ be a root automorphism which maps $f(\widetilde{\Pi})$ to $\widetilde{\Pi}^{\prime}$. Let $\sigma$ be a $\mathfrak{g}$-automorphism which stabilizes $\mathfrak{h}$, and for all $\alpha \in \Delta$,

$$
\sigma\left(\mathfrak{g}_{\alpha}\right)=\mathfrak{g}_{h(\alpha)} .
$$

By (5.22), (5.23) and (5.24), it follows that $\sigma(\mathfrak{m})=\mathfrak{m}^{\prime}$. This proves the proposition.

We illustrate the sliding process of Proposition 5.3 by Example 5.4 below.

Example 5.4 Figure 3a,b and strongly isomorphic Levi factors.

Let $c$ be the circling in Fig. 3a. Let $k$ be the Kac diagram given by

$$
k_{\alpha}=\left\{\begin{array}{l}
1 \text { if } \alpha \in c(\mathrm{D}), \\
0 \text { if } \alpha \in \mathrm{D} \backslash c(\mathrm{D}) .
\end{array}\right.
$$

By Theorem 1.1, $k$ represents a $\mathfrak{g}$-automorphism $\theta$ of order $\sum_{c(\mathrm{D})} a_{\alpha}=2+5+2=9$. Let $\omega=\exp (2 \pi i / 9)$.

Let the five rightmost vertices of Fig. 3a represent $\alpha_{0}, \alpha_{1}, \ldots, \alpha_{4} \in \Delta$ consecutively. The eigenvalues of $\theta$ on their root spaces are

$$
\left.\theta\right|_{\mathfrak{g}_{\alpha_{i}}}=(\omega, 1,1, \omega, 1) .
$$

Our slidable region $R \subset \mathrm{D}$ consists of $\left\{\alpha_{i}\right\}_{1}^{4}$. It is the Dynkin diagram of a subalgebra $\mathfrak{s l}(5, \mathbb{C}) \cong \mathfrak{g}_{R} \subset \mathfrak{g}$ with root system $\Delta_{R} \subset \Delta$ and Weyl group $W_{R} \subset$ aut $\left(\Delta_{R}\right)$. Write $\Delta_{R}=\left\{e_{i}-e_{j} ; i, j=1, \ldots, 5\right\}$ in the standard way, so that $W_{R} \cong S_{5}$ by its action on $\left\{e_{i}\right\}_{1}^{5}$. Consider $f=(14253) \in W_{R}$, namely $f\left(e_{1}\right)=e_{4}$ and so on. We have

$$
f\left(\alpha_{1}\right)=\alpha_{4}, f\left(\alpha_{2}\right)=-\alpha_{1}-\alpha_{2}-\alpha_{3}-\alpha_{4}, f\left(\alpha_{3}\right)=\alpha_{1}, f\left(\alpha_{4}\right)=\alpha_{2} .
$$

Here $W_{R}$ is generated by the reflections $\left\{r_{\alpha} ; \alpha \in \Delta_{R}\right\}$. By regarding $\alpha$ as a member of $\Delta$, each $r_{\alpha}$ extends naturally to aut $(\Delta)$, so $f \in \operatorname{aut}(\Delta)$. It satisfies

$$
f\left(\alpha_{0}\right)=\alpha_{0}+\alpha_{1}+\alpha_{2}+\alpha_{3}, f(\alpha)=\alpha \text { for all } \alpha \in \mathrm{D} \backslash R .
$$

Recall that the Kac diagram $k$ represents $\theta$ in the sense that the vertices of D represent some $\widetilde{\Pi}$ by (5.4), and $\left.\theta\right|_{\mathfrak{g}_{\alpha}}=\omega^{k_{\alpha}}$ for all $\alpha \in \widetilde{\Pi}$.

Suppose that we now let D represent $f(\widetilde{\Pi})$ instead. By (5.25), (5.26) and (5.27), for all $\alpha \in f(\widetilde{\Pi})=\mathrm{D}$,

$$
\left.\theta\right|_{\mathfrak{g}_{\alpha}}=\left\{\begin{array}{cl}
\left(\omega^{2}, 1, \omega^{-1}, 1,1\right) & \text { if } \alpha \in\left\{f\left(\alpha_{i}\right)\right\}_{0}^{4}, \\
\omega^{k_{\alpha}} & \text { if } \alpha \in \mathrm{D} \backslash\left\{f\left(\alpha_{i}\right)\right\}_{0}^{4} .
\end{array}\right.
$$

For example, (5.25) and (5.27) say that $\left.\theta\right|_{\mathfrak{g}_{f\left(\alpha_{0}\right)}}=\omega \cdot 1 \cdot 1 \cdot \omega=\omega^{2}$. Let $c^{\prime}$ be the circling where $\alpha \in \mathrm{D}$ is not circled if and only if $\left.\theta\right|_{\mathfrak{g}_{\alpha}}=1$. The vertices of $\mathrm{D} \backslash c^{\prime}(\mathrm{D})$ are roots of $\mathfrak{m}$. 
But (5.25) and (5.28) show that the subdiagrams $\mathrm{D} \backslash c^{\prime}(\mathrm{D})$ and $\mathrm{D} \backslash c(\mathrm{D})$ are equivalent. Since $\operatorname{Dyn}\left(\mathfrak{m}_{\mathrm{ss}}\right)=\mathrm{D} \backslash c(\mathrm{D})$, we have

$$
\operatorname{Dyn}\left(\mathfrak{m}_{\mathrm{ss}}\right)=\mathrm{D} \backslash c^{\prime}(\mathrm{D}) \text { with respect to } f(\widetilde{\Pi}) .
$$

By (5.28), $c^{\prime}$ is also Fig. 3b. Suppose that $\mathfrak{m}^{\prime}$ is represented by Fig. 3b, namely

$$
\operatorname{Dyn}\left(\mathfrak{m}_{\mathrm{ss}}^{\prime}\right)=\mathrm{D} \backslash c^{\prime}(\mathrm{D}) \text { with respect to some } \tilde{\Pi}^{\prime} .
$$

By repeating the construction of (5.24), we obtain a $\mathfrak{g}$-automorphism $\sigma$ such that $\sigma(\mathfrak{m})=$ $\mathfrak{m}^{\prime}$. Hence Fig. 3a,b represent strongly isomorphic Levi factors.

We shall see in the next section that the converse of Proposition 5.3 is false. Namely, $\mathfrak{m} \cong \mathfrak{m}^{\prime}$ does not imply that $c, c^{\prime}$ are related by sliding. We say that a circling $c$ is preferred if $1 \in\left\{a_{\alpha}\right\}_{c(\mathrm{D})}$. For example, Fig. 3a is not preferred because $\left\{a_{\alpha}\right\}_{c(\mathrm{D})}=\{2,5\}$.

Proposition 5.5 Every Levi factor $\mathfrak{m}$ can be represented by a preferred circling.

Proof Let $\mathfrak{m}$ be a Levi factor. In (3.2), we construct a preferred Kac diagram $k$, and ker $k=$ $\left\{\alpha \in \mathrm{D} ; k_{\alpha}=0\right\}$ are the simple roots of $\mathfrak{m}$. Let $c$ be the associated circling by (5.1). Then $c$ is a preferred circling which represents $\mathfrak{m}$.

Corollary 5.6 Let $c$ be a circling on $\mathrm{D}$ such that $\left\{a_{\alpha}\right\}_{c(\mathrm{D})}$ are relatively prime. There exists a preferred circling $c^{\prime}$ such that $\sum_{c^{\prime}(\mathrm{D})} a_{\alpha} \leq \sum_{c(\mathrm{D})} a_{\alpha}$, and $c, c^{\prime}$ represent strongly isomorphic Levi factors.

Proof We may assume that $\mathfrak{g}$ is exceptional, for otherwise $\left\{a_{\alpha}\right\}_{c(\mathrm{D})}$ are relatively prime if and only if they contain 1 . Let $c$ be a circling such that $\left\{a_{\alpha}\right\}_{c(\mathrm{D})}$ are relatively prime. By Theorems 1.2 and 1.3, $c$ represents a Levi factor $\mathfrak{m}$ in the sense of (5.3). We do not have a general construction for the desired circling $c^{\prime}$, but it is straightforward to check case by case. We discuss all the cases for $G_{2}, F_{4}, E_{6}$ and $E_{7}$ here.

$G_{2}$ : If $c$ is relatively prime but not preferred, then $c(\mathrm{D})=\{\gamma, \beta\}$ where $a_{\gamma}=2$ and $a_{\beta}=3$.

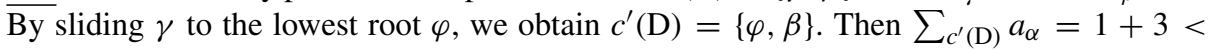
$2+3=\sum_{c(\mathrm{D})} a_{\alpha}$.

$F_{4}$ : We name the vertices as $v, w, x, y, z$ consecutively, where $v$ is the lowest root, and $\overline{v, w}, x$ are the long roots. The values of $a_{\alpha}$ are 1,2,3,4,2 respectively. If $c$ is relatively prime but not preferred, then $x \in c$ (D) and $v \notin c(\mathrm{D})$. If $w \in c(\mathrm{D})$, then we slide $w$ to $v$ and we are done. Hence we may assume that $w \notin c(\mathrm{D})$. If $y \in c(\mathrm{D})$, then we slide $x$ to $v$ and we are done. The only remaining case is $c(\mathrm{D})=\{x, z\}$. We first slide $z$ to $y$, then slide $x$ to $v$ and obtain $c^{\prime}(\mathrm{D})=\{v, y\}$. Consequently, $\sum_{c^{\prime}(\mathrm{D})} a_{\alpha}=a_{v}+a_{y}=1+4=3+2=a_{x}+a_{z}=$ $\sum_{c(\mathrm{D})} a_{\alpha}$.

$E_{6}$ : Let $c$ be a circling which is relatively prime but not preferred. Then there exists $\gamma, \beta \in$ $\overline{c(\mathrm{D})}$ such that $a_{\gamma}=2$ and $a_{\beta}=3$. Also, $\gamma$ is adjacent to a vertex $\delta$ with $a_{\delta}=1$ and $\delta \notin c$ (D). By sliding $\gamma$ to $\delta$, we obtained the desired $c^{\prime}$.

$E_{7}$ : Let $c$ be a circling which is relatively prime but not preferred. Then $c(\mathrm{D})$ contains $\beta$ $\overline{\text { such }}$ that $a_{\beta}=3$. Furthermore, we can assume that the two vertices adjacent to $\beta$ are not circled, for otherwise we can slide a vertex to the lowest root $\varphi$ and obtain $c^{\prime}$ immediately. With this observation, there are only four cases to consider, and they are listed in the left 
column of (5.29) below. In each case, we show that sliding leads to the desired circling $c^{\prime}$. Here • denotes circled vertex.

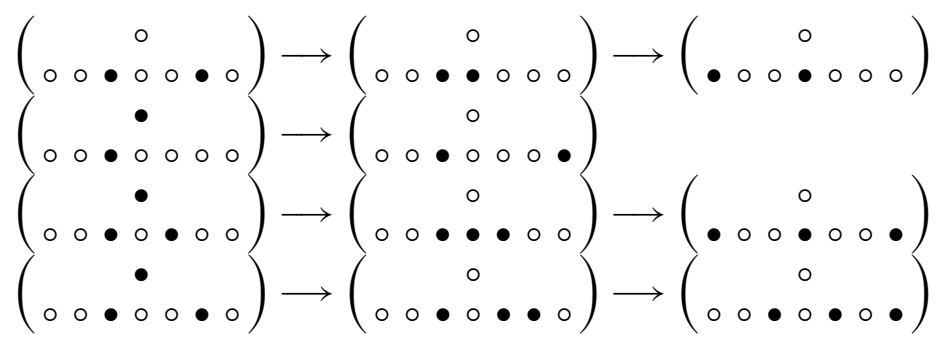

It is also straightforward to check all the cases of $E_{8}$ that a relatively prime circling $c$ leads to the desired circling $c^{\prime}$ by one of the following two methods:

(a) perform sliding (5.10) several times to obtain $c^{\prime}$,

(b) use Proposition 5.5 to construct $c^{\prime}$.

However, it is too lengthy to list all the cases of $E_{8}$ here. We provide some examples in the next section.

Proof of Theorem 1.4 Let $\theta$ be a finite order $\mathfrak{g}$-automorphism such that $\mathfrak{g}^{\theta}$ is a Levi subalgebra, represented by a Kac diagram $k$. It leads to a circling $c$ by (5.1), and $c$ represents $\mathfrak{g}^{\theta}$. By Theorems 1.2 and $1.3,\left\{a_{\alpha}\right\}_{c(\mathrm{D})}$ are relatively prime. Let $c^{\prime}$ be the circling given by Corollary 5.6. Let $k^{\prime}$ be the preferred Kac diagram

$$
k_{\alpha}^{\prime}=\left\{\begin{array}{l}
1 \text { if } \alpha \in c^{\prime}(\mathrm{D}), \\
0 \text { if } \alpha \in \mathrm{D} \backslash c^{\prime}(\mathrm{D}),
\end{array}\right.
$$

and let $\theta^{\prime}$ be the corresponding $\mathfrak{g}$-automorphism. Then $c^{\prime}$ represents $\mathfrak{g}^{\theta^{\prime}}$, and

$$
\begin{aligned}
\operatorname{order}\left(\theta^{\prime}\right) & =\sum_{c^{\prime}(\mathrm{D})} a_{\alpha} \text { by Theorem } 1.1 \text { and }(5.31) \\
& \leq \sum_{c(\mathrm{D})} a_{\alpha} \quad \text { by Corollary } 5.6 \\
& \leq \sum_{\mathrm{D}} a_{\alpha} k_{\alpha} \quad \text { by }(5.1) \\
& =\operatorname{order}(\theta) . \quad \text { by Theorem } 1.1
\end{aligned}
$$

By Corollary 5.6, $\mathfrak{g}^{\theta} \cong \mathfrak{g}^{\theta^{\prime}}$. The theorem follows.

\section{Examples}

In this section, we provide some examples of $E_{8}$ to discuss the process $c \rightsquigarrow c^{\prime}$ in Corollary 5.6. In particular, Fig. 4a,b illustrate (5.30)(a), and Fig. 4c,d illustrate (5.30)(b).

Recall that Fig. 3a leads to Fig. 3b by sliding. We draw Fig. 3a again as Fig. 4a here, and apply more steps of sliding (5.10) as follows. Here • denotes circled vertex.

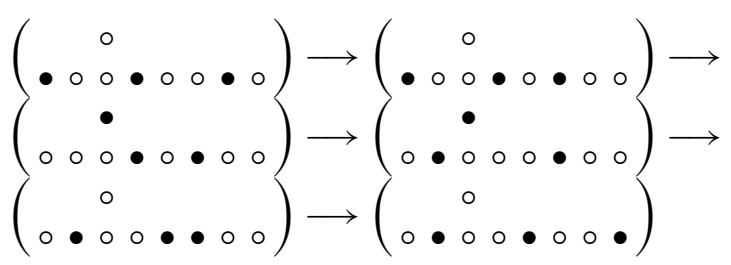




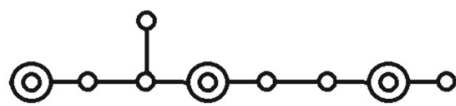

(a)

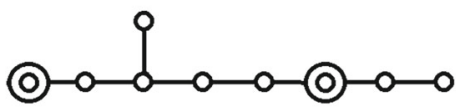

(c)

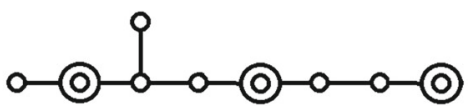

(b)

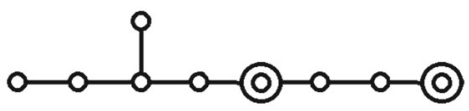

(d)

Fig. 4 Transformations to preferred circlings

They transform Fig. 4a to Fig. 4b. Denote them by $c, c^{\prime}$ respectively. By Proposition 5.3, they represent strongly isomorphic Levi factors. Also, $\sum_{c(\mathrm{D})} a_{\alpha}=\sum_{c^{\prime}(\mathrm{D})} a_{\alpha}=9$, and $c^{\prime}$ is preferred because $1 \in\left\{a_{\alpha}\right\}_{c^{\prime}(\mathrm{D})}$. Hence $c^{\prime}$ satisfies the conditions in Corollary 5.6.

Next we let $c$ be Fig. $4 \mathrm{c}$, and it represents $\mathfrak{m} \cong D_{5}+A_{2}+\mathbb{C}$ by (5.3). It has no sliding region, so (5.30)(a) does not work. By Proposition 5.5, there is a preferred circling $c^{\prime}$ which represents $\mathfrak{m}$. Since $c^{\prime}$ is preferred, the rightmost vertex has to be circled. The only way to circle another vertex such that $c^{\prime}$ represents $D_{5}+A_{2}+\mathbb{C}$ is indicated by Fig. $4 \mathrm{~d}$. We also have $\sum_{c(\mathrm{D})} a_{\alpha}=\sum_{c^{\prime}(\mathrm{D})} a_{\alpha}=5$, so $c^{\prime}$ satisfies the conditions in Corollary 5.6. This example illustrates (5.30)(b). It shows that we do not have sharp inequality on $\sum_{c^{\prime}(\mathrm{D})} a_{\alpha} \leq \sum_{c(\mathrm{D})} a_{\alpha}$. It also shows that the converse of Proposition 5.3 is false, namely sliding is a sufficient but not necessary condition for strong isomorphism.

Acknowledgements M. K. Chuah is supported by a grant from the Ministry of Science and Technology of Taiwan. The authors are very grateful to the referee for providing many helpful suggestions, and to I. Dimitrov for engaging in useful discussions. The authors wish to thank the Mathematics Departments of University of Bologna and National Tsing Hua University for providing warm hospitalities during their mutual visits.

Funding Open access funding provided by Alma Mater Studiorum - Università di Bologna within the CRUICARE Agreement.

Open Access This article is licensed under a Creative Commons Attribution 4.0 International License, which permits use, sharing, adaptation, distribution and reproduction in any medium or format, as long as you give appropriate credit to the original author(s) and the source, provide a link to the Creative Commons licence, and indicate if changes were made. The images or other third party material in this article are included in the article's Creative Commons licence, unless indicated otherwise in a credit line to the material. If material is not included in the article's Creative Commons licence and your intended use is not permitted by statutory regulation or exceeds the permitted use, you will need to obtain permission directly from the copyright holder. To view a copy of this licence, visit http://creativecommonshorg/licenses/by/4.0/.

\section{References}

1. Bourbaki, N.: Elements de mathematique. Groupes et algebres de Lie, Ch. IV-VI. Herman, Paris (1968)

2. Carmeli, C., Fioresi, R., Varadarajan, V.S.: Highest weight Harish-Chandra supermodules and their geometric realizations. Transf. Groups 25, 33-80 (2020)

3. Dimitrov, I., Fioresi, R.: On Kostant root systems for Lie superalgebras. J. Alg. 570, 678-701 (2021)

4. Dimitrov, I., Futorny, V., Grantcharov, D.: Parabolic sets of roots. Contemp. Math. 499, 61-74 (2009)

5. Ferrara, S., Marrani, A., Yeranyan, A.: Freudenthal duality and generalized special geometry. Phys. Lett. B 701, 640-645 (2011) 
6. Harish-Chandra: Representations of semi-simple Lie groups IV, V, VI. Amer. J. Math. 77, 743-777 (1955). no. 78, 1-41 and 564-628 (1956)

7. Kac, V.G. Infinite Dimensional Lie Algebras, 3rd edn. Cambridge Univ. Press, Cambridge (1990)

8. Knapp, W.A.: Lie Groups beyond an Introduction, 2nd edn., Progr Math, vol. 140. Birkhäuser, Boston (2002)

9. Kostant, B.: Root systems for Levi factors and Borel-de Siebenthal theory, in "Symmetry and Spaces", pp. 129-152, Progr. Math, vol. 278. Birkhäuser, Boston (2010)

10. Marrani, A.: Freudenthal duality in gravity: From groups of type $E_{7}$ to pre-homogeneous spaces. P-Adic Numbers. Ultrametric Anal. Applic. 7, 322-331 (2015)

Publisher's Note Springer Nature remains neutral with regard to jurisdictional claims in published maps and institutional affiliations. 